\title{
Cosmic-ray capabilities of the ARIANNA neutrino experiment
}

\author{
Anna Nelles* for the ARIANNA Collaboration \\ University of California, Irvine \\ E-mail: anelles@uci.edu
}

The ARIANNA experiment is designed to measure the radio emission created by neutrinos interacting in the Antarctic ice. The detection is achieved by installing high gain log-periodic dipole antennas just below the surface, which will record the radio pulses. The current design foresees more than 1000 independent radio detection stations with a spacing of at least a kilometer.

The ARIANNA design also includes upward facing antennas to reject radio signals from air showers. These rejection capabilities in turn make ARIANNA a very efficient cosmic ray detector. The bandwidth of $50-1000 \mathrm{MHz}$ is wider than those of most dedicated air shower arrays and the extremely radio-quiet environment on the Ross Ice Shelf allows for the detection of cosmic rays based purely on a self-trigger.

We will report on the cosmic ray detection conducted with the pilot-stage array. We will discuss self-trigger efficiency and event reconstruction including arrival directions, energy and flux measurements. These measurements illustrates that air shower signals are an excellent calibration tool for neutrino measurements and to monitor detector operations.

35th International Cosmic Ray Conference

10-20 July, 2017

Bexco, Busan, Korea

${ }^{*}$ Speaker. 


\section{Introduction}

A fair amount of experience has been gathered with the ARIANNA hexagonal array (HRA) and other set-ups to study the feasibility of the radio detection of neutrino interactions in the ice of Antarctica. Recent developments, such as establishing propagation properties of the ice [1] and new studies to search for neutrino signals [2], have shown that the detection technique is highly promising. A strong background is expected from the radio emission of air showers [3]. This is especially relevant for a detector, like ARIANNA, where the antennas are installed close to the ice surface, which means that the radio signals from air showers only have to propagate short distances in the ice, before reaching the detector. However, the ARIANNA design is equipped with unique features that will allow for an almost perfect identification of the cosmic ray signals and therefore makes ARIANNA also an efficient cosmic ray detector.

\section{Cosmic Ray Measurements with the HRA}

The ARIANNA HRA [4] was completed in 2014 and has been extended with three special purpose stations in the following years. The original HRA consists of seven stations on a hexagonal grid. Every station on the grid is equipped with four downward pointing logarithmic dipole antennas. The three special purpose stations have four differently aligned antennas, such as pointing upwards or tilted to the side (see also [5]). Regardless of the orientation, each antenna channel is read-out individually with a sampling rate of one or two Gigasamples per second, providing a bandwidth of up to $1 \mathrm{GHz}$. At every dual threshold trigger (high-low threshold, to suppress thermal fluctuations), waveforms of 256 samples are read-out. The data is stored locally and is also transmitted in real-time either via long-range wifi or satellite (Iridium SBD) to the server in the United States. The station run fully autonomous on solar power, with a planned extension to wind-power.

The stations with the upward pointing antennas are dedicated stations to establish the background of cosmic rays and other radio interference, and to use the data as reconstruction training set as they resemble the expected neutrino signal.

\subsection{Cosmic Ray Identification}

The ARIANNA event identification for both neutrinos and cosmic rays, relies on both the unique spectral properties of the expected signals and the unique behavior of the LPDAs [6]. The frequency content of the signal (both neutrinos and cosmic rays) is broadly peaked around a certain frequency and falls off smoothly. The strongest observable frequency depends on the distance to the location that corresponds to the Cherenkov angle, where in principle all frequencies are present. Air shower signals are generally stronger at lower frequencies (below $100 \mathrm{MHz}$ ) than from neutrino induced showers (between 100 and $500 \mathrm{MHz}$ ), due to the larger dimensions of the shower front. However, the frequency content most strongly depends on the distance to the Cherenkov angle. Combining the characteristic signal spectra with the dispersiveness of the LPDA leads to characteristically chirping pulses as shown in Figure 1. Using simulated predictions of these pulses as search templates in a cross-correlation study has allowed us to identify cosmic rays with high precision (see Figure 2). 

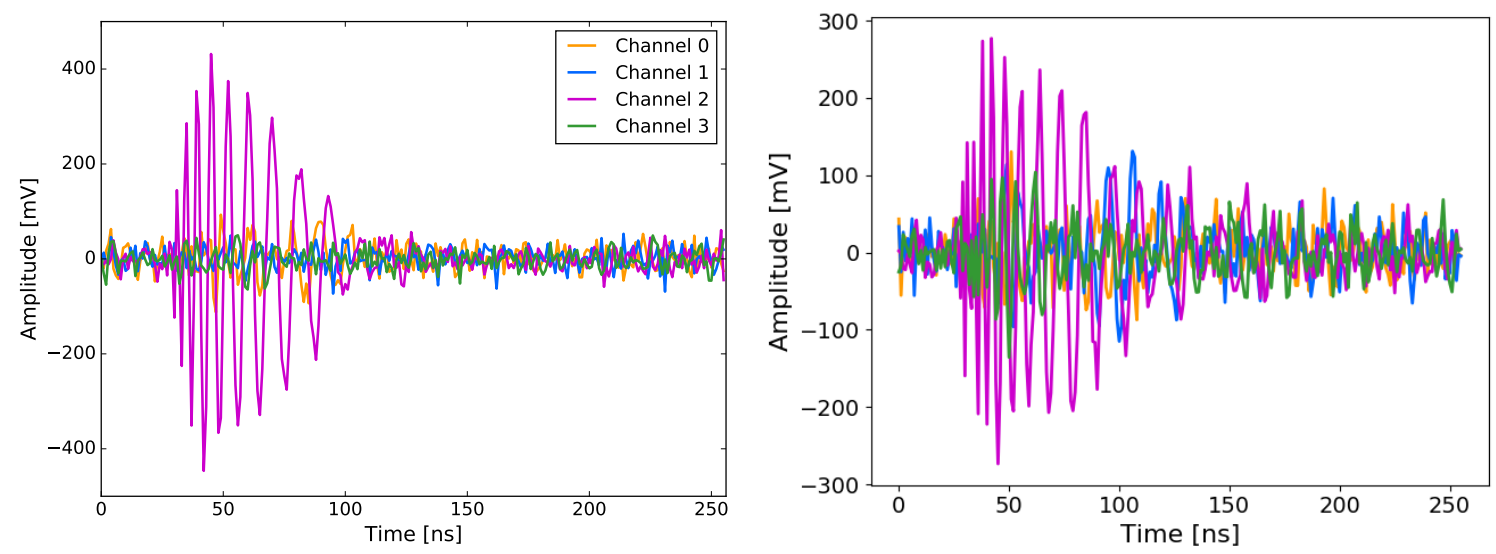

Figure 1: Left: Typical simulated template of a cosmic ray signal for one of the dedicated cosmic ray stations. Right: Measured cosmic ray signal in season 2016.
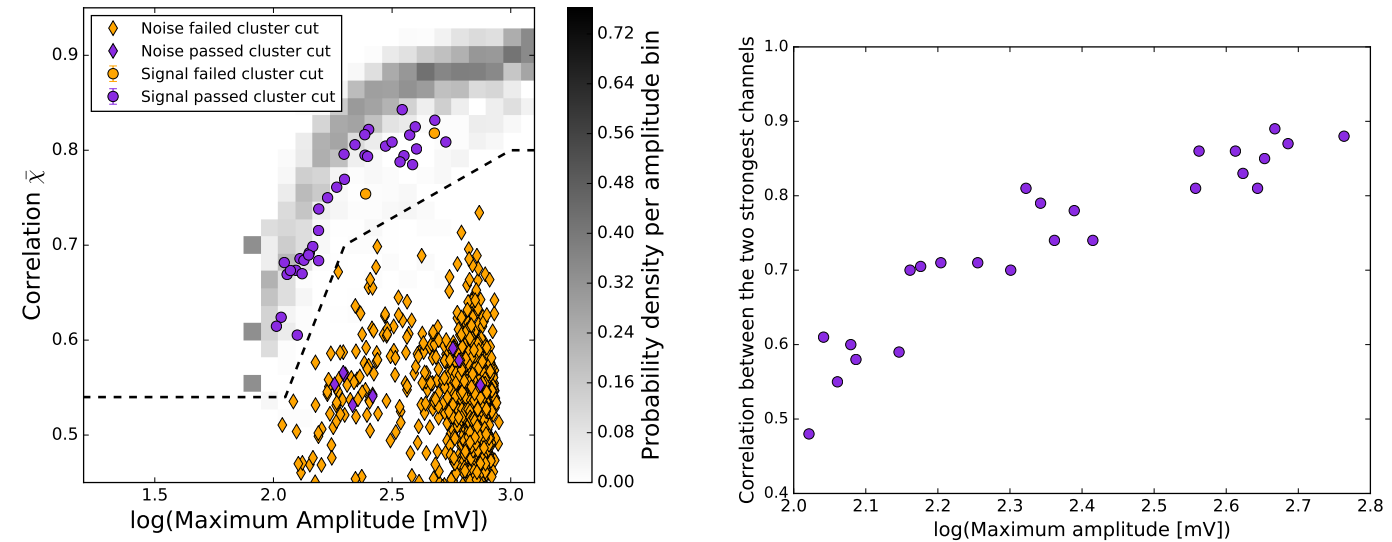

Figure 2: Left: Overview of the procedure to identify cosmic ray signals in one of the dedicated cosmic ray stations. Shown is the average correlation of the waveform with all signal templates (simulated air shower signals) as function of maximum signal amplitude. The grey background shows the predicted signal space from simulations, while the markers indicate the measured data. The dashed line separates cosmic rays from background. This cut retains more than $99 \%$ of the signal. For more details see [3]. Right: The influence of noise on the correlation. Shown is the correlation between two parallel antenna channels as function of maximum signal amplitude of detected cosmic rays. The data is from a new dedicated station that has four upward facing channels. The figure shows that the signal amplitude dominates the correlation. Given that the noise is almost constant at the ARIANNA site and in the relevant bandwidth, this shows that the influence of the noise dominates the correlation, as predicted from simulations. This result gives additional confidence in the quality of the simulations. 
The identified cosmic rays are clearly separated from the otherwise recorded noise (see Figure 3). In 2016, a more sensitive station has been deployed to record cosmic rays with four upward facing antennas, which allows us to better test reconstruction algorithms. Using the correlation to a single cosmic ray template (rather than the more stable average correlation value $\bar{\chi}[3]$ ), already gives an indication that the station is much more sensitive to cosmic rays (see Figure 4). However, also more periods of high correlating noise can be seen, which will still have to be studied in detail. Also, the data has not been corrected for the temperature dependent gain of the amplifiers. The quality of the measured signals has also improved as discussed in Figure 7.

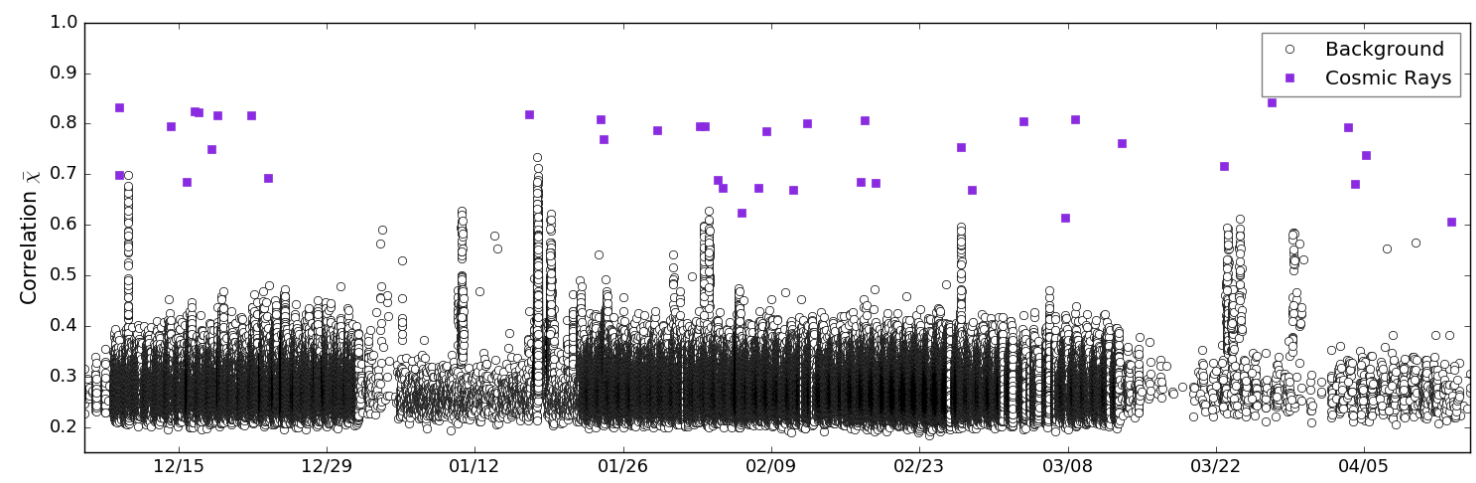

Figure 3: Event selection of the 2015/2016 data as discussed in [3]. The figure shows the random arrival times of the cosmic rays. In January 2016 the trigger had been changed, which suppressed cosmic rays.

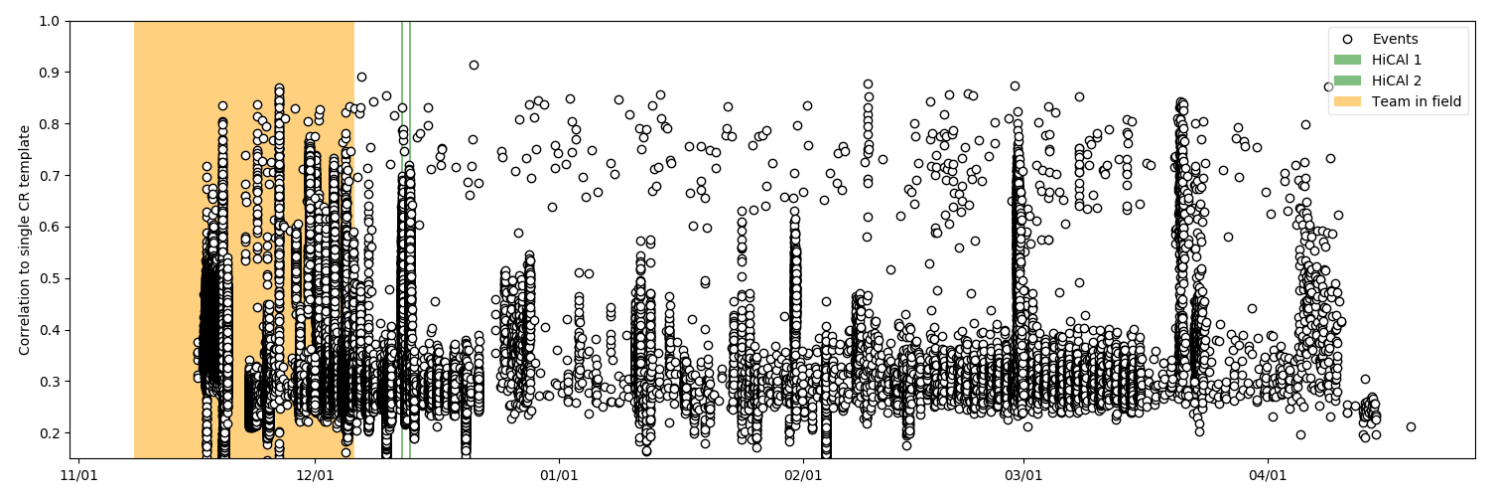

Figure 4: Overview of the data recorded of one of the dedicated cosmic ray stations in the polar season of 2016/2017. The event rates have not been corrected for live-time or trigger threshold. As opposed to the data shown in Figure 3, this station has four instead of two upward facing antennas, which makes it much more efficient for cosmic rays, but also more sensitive to pulses induced by storms or human activity in the field. The station also clearly recorded the flights of HiCal [7]. HiCal is a balloon-borne pulser that was launched by the ANITA Collaboration in January 2017.

\subsection{Cosmic Ray Flux}

Based on a thorough analysis of all cosmic rays recorded in the season of 2015/16, a first flux measurement of cosmic rays has been established from ARIANNA data. The agreement between 
ARIANNA and world-data, as shown in Figure 5, provides confidence in the understanding of our detector and the detected signals. With a larger array we will be able to significantly decrease the uncertainties and provide a competitive flux measurement. The analysis, as presented in [3] is currently limited by only 32 recorded events and large uncertainties on the energy reconstruction.

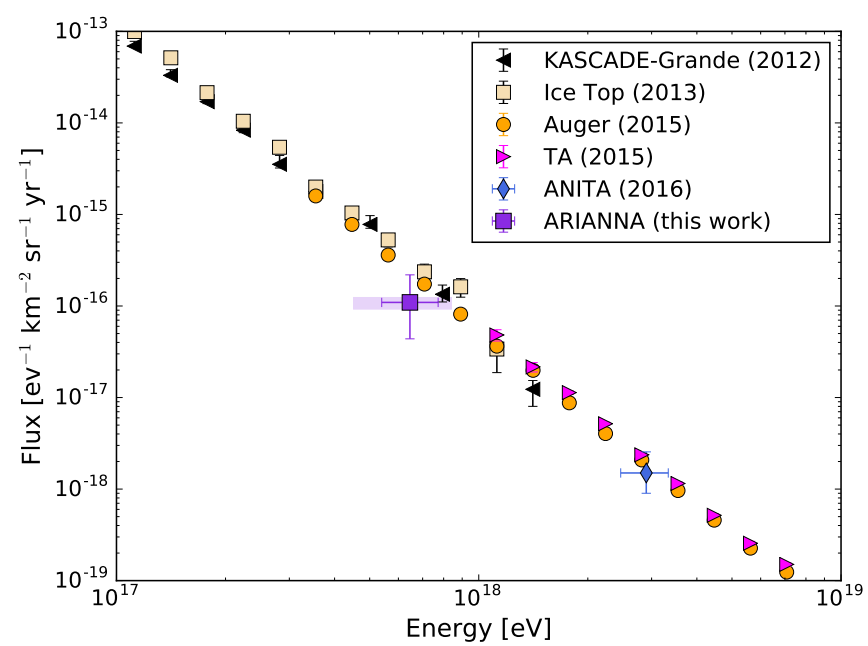

Figure 5: Flux of cosmic rays as function of energy. The data from the HRA as derived in this work is shown together with published flux data from KASCADE-Grande [8], the Pierre Auger Observatory [9], Telescope Array [10], IceTop [11] and ANITA [12]. The errorbars indicate the uncertainty on the mean energy as well as the exposure. The uncertainty on the scale of the energy is indicated by the shaded area.

\subsection{Cosmic Ray Properties}

One of the key characteristics of an air shower is the energy of the primary cosmic ray. As shown in [13] by the Auger Collaboration, a reconstruction based on radio signals has the potential to be as accurate as the best known energy measurements. At a station spacing of at least $1 \mathrm{~km}$, an ARIANNA-like detector will typically only detect air showers in one or two stations at the same time. This requires a different approach than used by dedicated air shower experiments that use an LDF-like approach such as in $[13,14]$.

We have shown in [3] that the frequency spectrum can be used to estimate the energy of a cosmic ray (see also Figure 6). Validating this method has been hampered by too few measured events and the fact that only one spectrum had been measured per shower. With the additional data taken with the new set-up and the improved data quality with two parallel channels (see Figure 7), the true potential of this method will be studied. A dedicated method of energy reconstruction that can also be used to determine the energy of the recorded neutrino shower, is currently being developed. The ARIANNA data is very suitable for a method based on the frequency slope, due to two reasons: the band-width of the system up to $1 \mathrm{GHz}$ is much wider than the typical air shower experiment, which provides a better fitting space for a spectral analysis. Also, the dispersive characteristics of the LPDA smear the pulse so that each can be measured with several sampling points, which increases the frequency resolution of the pulse opposed to non dispersive systems, where the pulse is only measured in very few samples. 

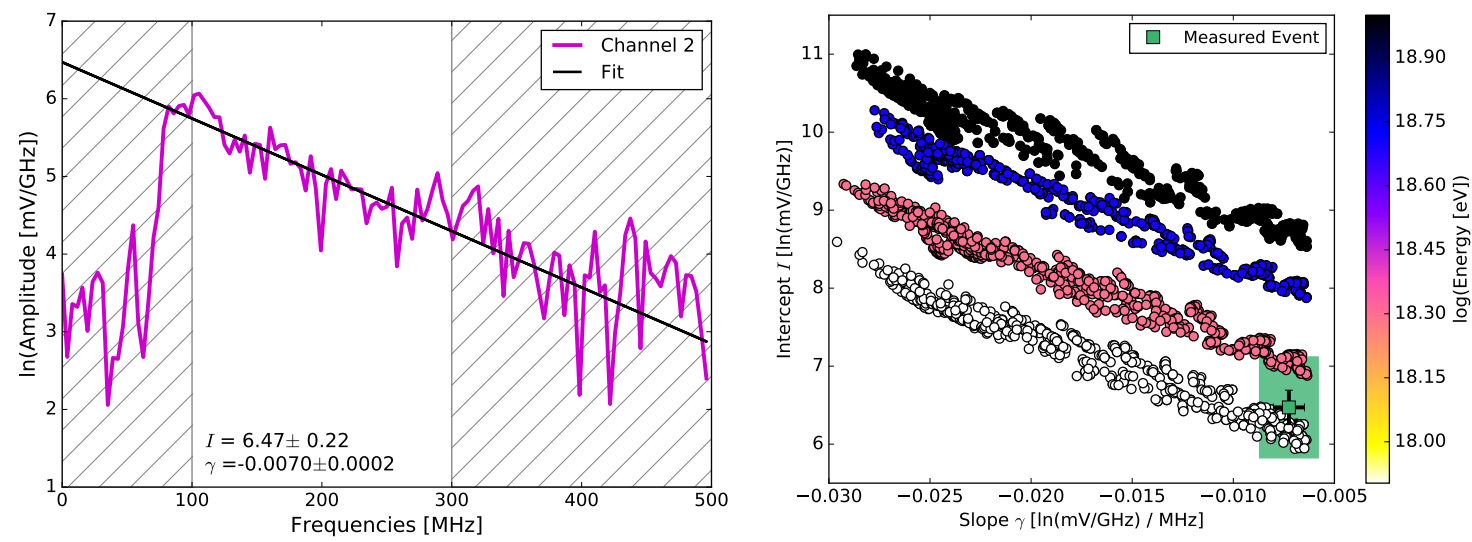

Figure 6: Left: Measured frequency spectrum of a cosmic ray signal, fitted with a straight line between 100 and $300 \mathrm{MHz}$. Right: A scatter plot showing the slope and intercept of these lines for simulated events of different energies. The circles show simulated air showers fitted with the same procedure, while the green square indicates the measurement (left) with uncertainties. For more details see [3].
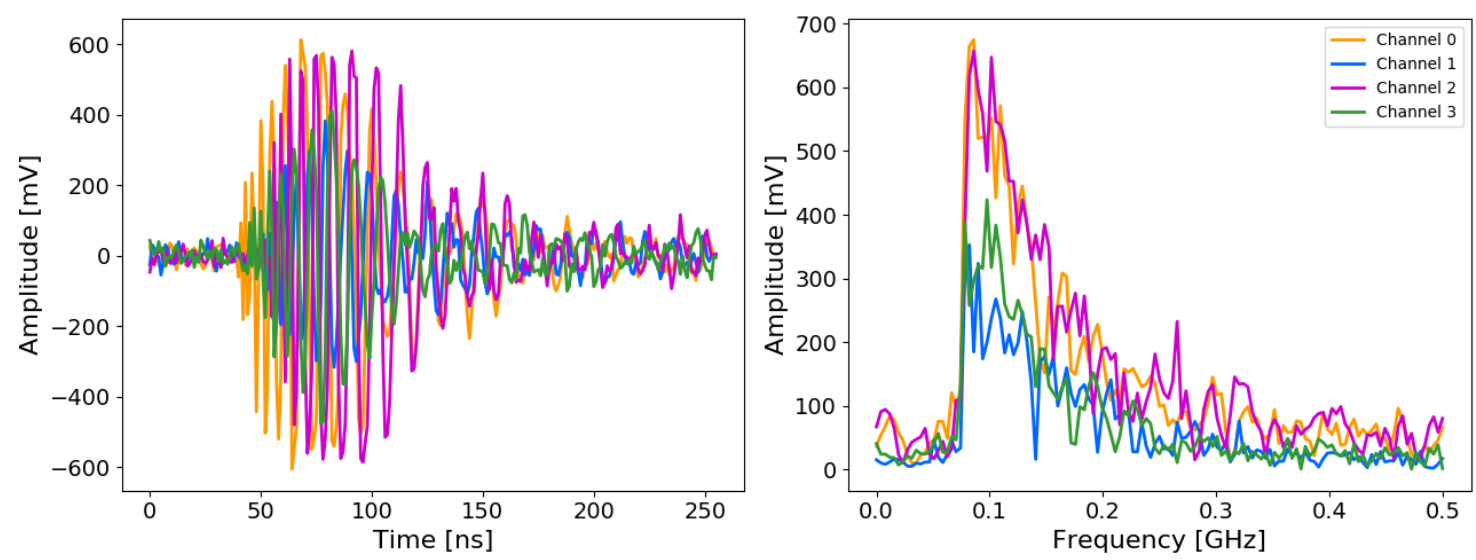

Figure 7: Event as recorded with a dedicated cosmic ray station in 2017. The channel pairs $(0,2)$ and $(1,3)$ belong to parallel antennas. The pairs are orthogonal to each other. Shown is the amplitude calibrated data.

\section{Capabilities of a large ARIANNA-like neutrino array}

We discuss in [2] the capabilities of an ARIANNA-like array with respect to neutrino detection. The location that is ultimately chosen for the array (Moore's Bay on the Ross Ice Shelf, South Pole or another location) and its ice properties will determine the number and spacing of stations needed to reach the first design goal of $10^{-9} \mathrm{GeV} \mathrm{cm}^{-2} \mathrm{~s}^{-1} \mathrm{sr}^{-1}$. These boundary conditions will ultimately determine the layout of the detector. All stations will need upward facing antennas to be able to fully veto cosmic ray signals. This is because air shower signals resemble neutrino induced signals and only the up-to-down ratio of the directional LPDAs will be a high-efficiency tool for distinction. With the upward facing antennas, however, the array will also be a capable cosmic ray detector.

Figure 8 shows the estimated rate of detected cosmic rays for an ARIANNA-like array with 


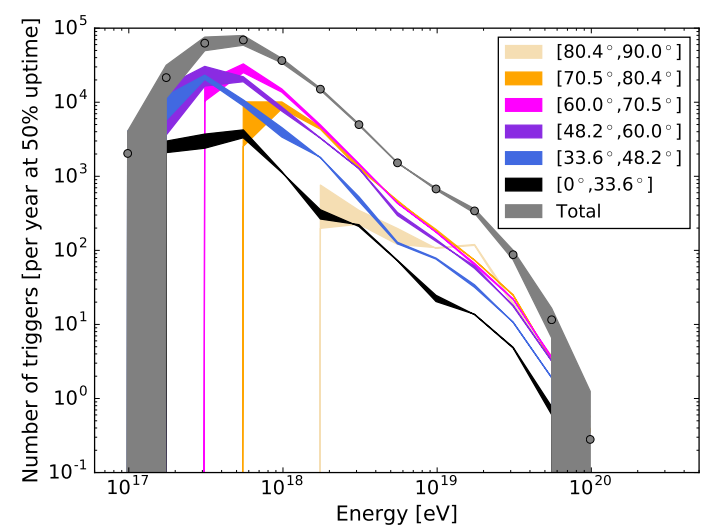

Figure 8: Right: Predicted cosmic rays triggers for an ARIANNA-like array with 1296 stations in Antarctica. Shown are half-decade energy bins. The predictions are based on the cosmic ray flux as measured by the Pierre Auger Collaboration [9].

1296 autonomous stations, with four upward facing antennas at a spacing of $1 \mathrm{~km}^{2}$. The trigger is set to be about $4 \sigma$ above the noise level in two out of four antennas. The trigger criteria are currently freely adjustable, also with respect on which antenna needs to record a signal. At a spacing of $1 \mathrm{~km}^{2}$ most events are already only detected in one station at the same time, which is sufficient for reconstruction. This means that increasing the spacing can only increase the number of triggers, however, only marginally. As all stations are independent, the number of detection scales with lifetime and number of stations. All such changes will not affect the capabilities as cosmic ray array and can be freely optimized for neutrino detection.

Reducing the number of upward facing antennas, will still allow to veto all cosmic ray events, but will reduce the number of triggers, if a threshold crossing in two antennas is required. With only two antennas, however, also the reconstruction of the arrival direction will no longer be possible, as this requires at least three measurements. Without arrival direction, also other parameters, such as energy and possibly the height of the shower maximum remain unobtainable. Consequently, the only design requirement to keep the cosmic ray capabilities is the number of upward facing antennas to at least three.

With an expected measurement of at least 5 years and at least 300 stations, an ARIANNA-like array will likely detect cosmic rays up to $10^{20} \mathrm{eV}$ and can provide an independent measurement of energy spectrum and possibly composition. With expected angular resolution of one degree, the array is also suitable to search for anisotropies.

\section{Conclusions}

An ARIANNA-like array using LPDAs installed close to the surface of the ice will not only be a promising radio neutrino detector, but also an efficient cosmic ray array. We have shown that cosmic rays can easily be identified using the data from the already installed HRA. The measured flux agrees with world data. The predicted event rates for a large array cover the energy range from the detection threshold at about $10^{17} \mathrm{eV}$ up to the cut-off in the cosmic ray spectrum. 


\section{Acknowledgements}

We wish to thank the US National Science Foundation for its support of the ARIANNA project under grant PHY-1607719. We also acknowledge support from the National Research Nuclear University MEPhI (Moscow Engineering Physics Institute) and from the German Research Foundation (DFG, grant NE 2031/1-1). We are grateful for the outstanding cooperation in field planning and operations by the Antarctic Support Contractor.

\section{References}

[1] S.W. Barwick for the ARIANNA Collaboration, Optimization of ARIANNA station configuration, These proceedings, PoS(ICRC2017)1042.

[2] C. Persichilli for the ARIANNA Collaboration, Radio search for EHE neutrinos with the ARIANNA pilot array, and ARIANNA performance projections, These proceedings, PoS(ICRC2017)977.

[3] S. W. Barwick, D. Z. Besson, A. Burgman, et al., Radio detection of air showers with the ARIANNA experiment on the Ross Ice Shelf, Astroparticle Physics 90 (2017) 50-68.

[4] S. W. Barwick, E. C. Berg, D. Z. Besson, et al., Design and Performance of the ARIANNA HRA-3 Neutrino Detector Systems, IEEE Transactions on Nuclear Science 62.

[5] S.H. Wang for the ARIANNA Collaboration, Calibration, Performance, and Cosmic Ray Detection of ARIANNA-HCR Prototype Station, These proceedings, PoS(ICRC2017)358.

[6] S. W. Barwick, E. C. Berg, D. Z. Besson, et al., Time-domain response of the ARIANNA detector, Astroparticle Physics 62 (2015) 139-151.

[7] P. W. Gorham, P. Allison, O. Banerjee, et al., Antarctic Surface Reflectivity Measurements from the ANITA-3 and HiCal-1 Experiments, Journal of Astronomical Instrumentation 6 (2017) 1740002-213.

[8] W. D. Apel, J. C. Arteaga-Velázquez, K. Bekk, et al., The spectrum of high-energy cosmic rays measured with KASCADE-Grande, Astroparticle Physics 36 (2012) 183-194.

[9] The Pierre Auger Collaboration, A. Aab, P. Abreu, et al., The Pierre Auger Observatory: Contributions to the 34th International Cosmic Ray Conference (ICRC 2015), ArXiv e-prints 1509.03732.

[10] T. Abu-Zayyad, R. Aida, M. Allen, et al., Energy spectrum of ultra-high energy cosmic rays observed with the Telescope Array using a hybrid technique, Astroparticle Physics 61 (2015) 93-101.

[11] M. G. Aartsen, R. Abbasi, Y. Abdou, et al., Measurement of the cosmic ray energy spectrum with IceTop-73, Physical Review D 88 (4) (2013) 042004.

[12] H. Schoorlemmer, K. Belov, A. Romero-Wolf, et al., Energy and flux measurements of ultra-high energy cosmic rays observed during the first ANITA flight, Astroparticle Physics 77 (2016) 32-43.

[13] A. Aab, P. Abreu, M. Aglietta, et al., Measurement of the Radiation Energy in the Radio Signal of Extensive Air Showers as a Universal Estimator of Cosmic-Ray Energy, Physical Review Letters 116 (24) (2016) 241101.

[14] A. Nelles, S. Buitink, A. Corstanje, et al., The radio emission pattern of air showers as measured with LOFAR - a tool for the reconstruction of the energy and the shower maximum, JCAP 5 (2015) 018. 